\title{
Ärztliche Kandidatinnen und Kandidaten für den National- und Ständerat stellen sich vor
}

\section{Fribourg}

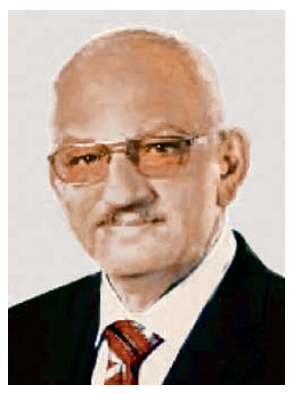

Michel Zadory, 1948, Estavayer-le-Lac

Spécialiste en chirurgie orthopédique FMH, médecin-chef du HIB

Député au Grand Conseil FR, conseiller communal à Estavayer-le-Lac

Candidat UDC au Conseil national
Le maintien de l'obligation de contracter, la défense des assurés et des malades, la lutte contre la pénurie des médecins de premier recours, la défense des intérêts du corps médical et des professions paramédicales sont autant de thèmes que nous devons soutenir avec vigueur. Le lobby des médecins est trop faible actuellement aux chambres fédérales.

A nous de mouiller notre blouse blanche pour défendre notre corporation.

\section{Jura}

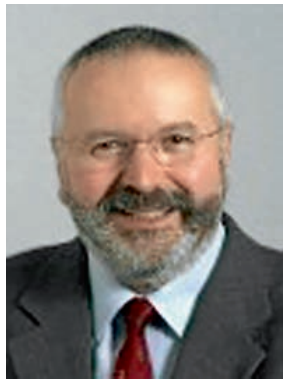

Dominique Baettig, 1953, Delémont

Médecin spécialiste en psychiatrie FMH

Candidat UDC au Conseil national

Envie non seulement de défendre le corps médical contre la double mainmise de l'Etat bureaucratique et celle des assurances qui jouent les deux sur les peurs, mais aussi l'usager payeur de primes de plus en plus insupportables qui servent à «traiter» les problèmes de société insolubles qu'on psychiatrise. Je m'inquiète des dérives coûteuses de la médecine étatique et technique qui s'éloigne de plus en plus du rapport direct de service rendu, des soins nécessaires, adéquats et appropriés pour fournir des prestations (auxquelles on accède de manière aléatoire ou injuste en fonction des circonstances et des personnalités) à consommer passivement, qu'on incite à utiliser sans se préoccuper de la motivation réelle à s'engager dans un comportement de participation responsable.

\section{Luzern}

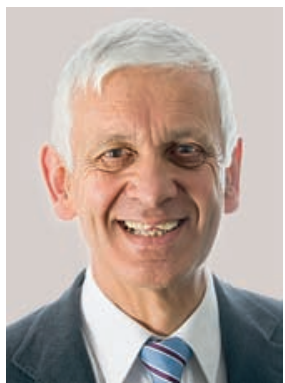

\section{Walter Häcki, 1942, Luzern}

Facharzt für Gastroenterologie und Innere Medizin FMH,

freipraktizierend mit Belegarzttätigkeit an der Klinik St. Anna, Luzern. 1979-1992 Leiter Gastroenterologie, Medizinische Klinik, Universitätsspital Zürich. Seit 12 Jahren Grossrat im Kanton Luzern.

Nationalratskandidat der SVP
Ich denke wirtschaftspolitisch liberal, in den Werten konservativ. Wir Ärzte sehen den Patienten und seine Ängste im Zentrum unserer Arbeit. Dazu gehören zwingend die freie Arzt- und Spitalwahl als wichtigste Qualitätssicherung. Die Politik hat sich leider immer mehr davon entfernt, der nächste Schritt sind die SwissDRG, die erstmals den Kodierer zur wichtigsten Person im Spital machen und nicht mehr den Arzt - er ist nur noch Kostenfaktor.

Wir brauchen gute und genügend Praxisärzte. Jeder arbeitet effizienter und kostengünstiger als Ambulatorien. Die FMH hat leider in den letzten Jahren die Misere der Ärzte aktiv und passiv mitverschuldet. Wir brauchen niedergelassene Ärzte im eidgenössischen Parlament. Nur wer in Kommissionen sitzt, kann mitbestimmen. 


\section{Ticino}

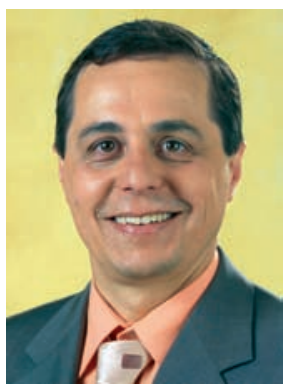

Ignazio Cassis, 1961, Montagnola (Collina d'Oro)

Specialista in prevenzione e salute pubblica FMH

e specialista in medicina interna FMH

Consigliere nazionale

Candidato al Consiglio nazionale del Partito Liberale Radicale

\section{Siamo troppo pochi in Parlamento!}

Tutto cambia, ma non per forza in meglio. Perciò dobbiamo agire! La nostra bella professione è minacciata dalle mire espansionistiche di numerosi altri attori - sanitari e non - che negli ultimi anni sono venuti a sedersi alla stessa tavola.

La pressione esercitata dagli assicuratori diventa sempre più sproporzionata e vessatoria. D'altro canto i pazienti sono sempre meno pazienti e hanno sempre più aspettative. In mezzo noi, che giornalmente cerchiamo di salvare capra e cavoli. La gente ha molta fiducia in noi medici: abbandoniamo perciò il nostro proverbiale pudore e impegniamoci anche in politica, per costruire il mondo futuro. Solo così potremo garantire a tutti una medicina di qualità a costi sostenibili e a noi una professione gratificante.

\section{Ticino}

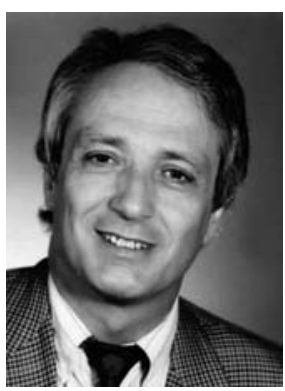

Franco Cavalli, 1942, Ascona

Professore spec. oncologiaematologia FMH, Direttore Istituto Oncologico della Svizzera Italiana

Candidato al Consiglio degli Stati per il PS

Se fossi eletto continuerei, come ho sempre fatto già al Consiglio nazionale, a battermi per continuare ad avere una sanità d'alta qualità e per un aumento degli aiuti federali alla ricerca, particolarmente nel settore biomedico. Attualmente il più grande pericolo per il nostro sistema di sanità è rappresentato dalla tendenza predominante del parlamento, ma specialmente del consigliere federale Couchepin, di voler a tutti costi deregolamentare il cosiddetto mercato sanitario. Questo in base all'ideologia che dice che più concorrenza porta ad una diminuzione dei costi. Ma l'esperienza internazionale dimostra che così facendo si ottiene esattamente l'effetto opposto. Ciò che però con sicurezza capita è che a dominare il sistema saranno poi le casse malati. È in questo senso che io mi sono sempre battuto contro l'abolizione dell'obbligo di contrarre.

\section{Zürich}

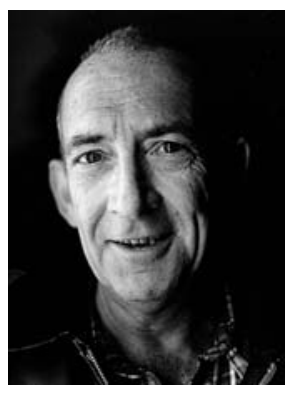

David Winizki, 1948, Zürich

Facharzt für Allgemeinmedizin FMH

Nationalratskandidat

der Alternativen Liste

Als Hausarzt, Stadtarzt bin ich konfrontiert mit der wachsenden Verarmung breiter Bevölkerungsschichten, knauserigeren Sozialversicherungen, omnipotenteren Krankenkassen, aber auch mit schwierigeren eigenen Lebensbedin- gungen - und all dies in einem Land, in dem eine Minderheit buchstäblich im Geld schwimmt. Je grösser die Unterschiede zwischen Arm und Reich sind, desto unfriedlicher und morbider wird eine Gesellschaft. Umverteilung tut not!

Das Gesundheitswesen muss der ganzen Bevölkerung ohne Rationierungen zugänglich sein und solidarisch, das heisst im Verhältnis zur ökonomischen Potenz finanziert werden. Leistungserbringer und Leistungserbringerinnen sollen gut aus-, weiter- und fortgebildet werden, vernetzt arbeiten und adäquat bezahlt werden.

Der Erhaltung der Gesundheit muss vermehrt Wert zugemessen werden mittels besserer, eine menschenwürdige Existenz sichernder Lebensbedingungen.

\section{Zürich}

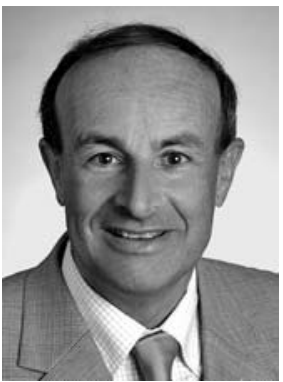

\section{Oskar Denzler, 1950, Winterthur}

Facharzt für Allgemeinmedizin FMH, Kantonsrat

Nationalratskandidat der FDP

«Ihr Hausarzt für Bern» mein Motto. Liberalen Grundwerten verpflichtet, engagiere ich mich für ein leistungsfähiges, finanzierbares und patienten- gerechtes Gesundheitswesen. Als Hausarzt liegt mir die Sicherstellung der ambulanten Grundversorgung mit attraktiven Arbeitsbedingungen am Herzen. Eine praxisnahe Ausbildung mit universitärer Verankerung gehört dazu. Der Standort Zürich für Forschung und Spitzenmedizin ist zu fördern. Persönliches Engagement und Unternehmertum in unserem Beruf muss sich lohnen und stellt die Basis für eine erfolgreiche Zukunft des Arztberufes dar. Zur Gesundheit gehört eine intakte Umwelt, der es Sorge zu tragen gilt. Die Ärzteschaft braucht eine starke Vertretung in Bern, wenn nicht andere über unsere $\mathrm{Zu}$ kunft bestimmen sollen. 